\title{
$(\theta, \phi)$-DERIVATIONS AS HOMOMORPHISMS OR AS ANTI-HOMOMORPHISMS ON A NEAR RING
}

\author{
ASMA ALI, HOWARD E. BELL AND REKHA RANI
}

\begin{abstract}
Let $N$ be a near ring. An additive mapping $d: N \longrightarrow N$ is said to be a $(\theta, \phi)$ derivation on $N$ if there exist mappings $\theta, \phi: N \longrightarrow N$ such that $d(x y)=\theta(x) d(y)+$ $d(x) \phi(y)$ holds for all $x, y \in N$. In the context of 3-prime and 3-semiprime nearrings, we show that for suitably-restricted $\theta$ and $\phi$, there exist no nonzero $(\theta, \phi)$-derivations which act as a homomorphism or an anti-homomorphism on $N$ or a nonzero semigroup ideal of $N$.
\end{abstract}

\section{Introduction}

Throughout the paper $N$ will be a zero-symmetric right nearring with multiplicative centre $Z$; and for arbitrary $x, y \in N,[x, y]$ will denote the commutator $x y-y x$. The nearring $N$ is said to be 3-prime if $x N y=\{0\}$, for $x, y \in N$ implies that $x=0$ or $y=0$ and 3-semiprime if $x N x=\{0\}$ implies that $x=0$; moreover, $N$ is called reduced if $N$ contains no nonzero nilpotents. A nonempty subset $A$ of $N$ is called a semigroup ideal if $A N \subseteq A$ and $N A \subseteq A$.

An additive mapping $d: N \longrightarrow N$ is said to be a derivation on $N$ if $d(x y)=x d(y)+d(x) y$ for all $x, y \in N$. Or equivalently ( $\operatorname{cf}[5]) d(x y)=d(x) y+x d(y)$ for all $x, y \in N$. As in [1], an additive mapping $d: N \longrightarrow N$ is called a $(\theta, \phi)$-derivation if there exist mappings $\theta, \phi: N \longrightarrow N$ such that $d(x y)=\theta(x) d(y)+d(x) \phi(y)$ for all $x, y \in N$. If $I$ denotes the identity map on $N$, an $(I, I)$-derivation is just a derivation, so the notion of $(\theta, \phi)$-derivation generalizes that of derivation. Moreover, the generalization is not trivial, as the following example shows:

Example 1. Let $N_{1}$ be an arbitrary zero-symmetric nearring; and $N_{2}$ be an algebra over a field of characteristic not 2, with basis $\{u, v, w\}$ and multiplication given by $u v=w$ and all other two-products of basis elemants equal to 0 . Let $N=N_{1} \oplus N_{2}$. Define $\theta, \phi: N \longrightarrow N$ by $\theta\left(\left(x_{1}, x_{2}\right)\right)=\left(x_{1}, 0\right)$ and $\phi\left(\left(x_{1}, x_{2}\right)\right)=\left(x_{1}^{2}, 0\right)$; and let $d: N \longrightarrow N$ be given by $d\left(\left(x_{1}, x_{2}\right)\right)=$ $\left(0, h\left(x_{2}\right)\right)$, where $h: N_{2} \longrightarrow N_{2}$ is the additive map such that $h(u)=u, h(v)=v$, and $h(w)=0$. It is easy to verify that $d$ is a $(\theta, \phi)$-derivation which is not a derivation.

Corresponding author: Asma Ali.

2010 Mathematics Subject Classification. 16Y30, 16W25.

Key words and phrases. 3-prime nearrings, 3 -semiprime nearrings, derivations, $(\theta, \phi)$-derivations. 
In [4] it is proved that if $R$ is a semiprime ring and $d$ is a derivation on $R$ which is either an endomorphism or an anti-endomorphism on $R$, then $d=0$. Of course derivations which are not endomorphisms or anti-endomorphisms on $R$ may behave as such on certain subsets of $R$; for example, any derivation $d$ behaves as the zero endomorphism on the subring $C$ consisting of all constants (i.e. elements $x$ for which $d(x)=0$.) In fact, in a semiprime ring, $d$ may behave as an endomorphism on a proper ideal of $R$. However, as noted in [4], the behaviour of $d$ is somewhat restricted in the case of a prime ring.

Recently the authors in [2] considered $(\theta, \phi)$-derivations $d$ acting as homomorphisms or anti-homomorphisms on a nonzero Lie ideal of a prime ring such that $u^{2} \in U$ for all $u \in U$ and concluded that $d=0$. The purpose of the present paper is to establish similar results in the setting of semigroup ideal of 3-prime and 3-semiprime nearrings admitting a $(\theta, \phi)$ derivation.

Usually, we shall require that $\theta(N)=N$, and that $\theta(a b)=\theta(a) \theta(b)$ and $\phi(a b)=\phi(a) \phi(b)$ for all $a, b \in N$. A $(\theta, \phi)$-derivation with $\theta$ and $\phi$ satisfying these conditions will be called a standard $(\theta, \phi)$-derivation.

\section{Preliminary results}

We begin with the following Lemma that was proved by the second author in [3].

Lemma 2.1. Let $N$ be a 3-prime nearring and A be a nonzero semigroup ideal of $N$.

(i) If $x \in N$ and $x A=\{0\}$ or $A x=\{0\}$, then $x=0$.

(ii) If $x, y \in N$ and $x A y=\{0\}$, then $x=0$ or $y=0$.

(iii) If $x \in N$ and $x$ centralizes $A$, then $x \in Z$.

Lemma 2.2. Let $N$ be an arbitrary nearring. If $d$ is a standard $(\theta, \phi)$-derivation on $N$, then $N$ satisfies the following partial distributive law:

$$
a(\theta(b) d(c)+d(b) \phi(c))=a \theta(b) d(c)+a d(b) \phi(c) \text { for all } a, b, c \in N .
$$

Proof. For $a, b, c \in N$, we have

$$
\begin{aligned}
d((a b) c) & =\theta(a b) d(c)+d(a b) \phi(c) \\
& =\theta(a) \theta(b) d(c)+(\theta(a) d(b)+d(a) \phi(b)) \phi(c) \\
& =\theta(a) \theta(b) d(c)+\theta(a) d(b) \phi(c)+d(a) \phi(b) \phi(c) \text { for all } a, b, c \in N .
\end{aligned}
$$

Again

$$
\begin{aligned}
d(a(b c)) & =\theta(a) d(b c)+d(a) \phi(b c) \\
& =\theta(a)(\theta(b) d(c)+d(b) \phi(c))+d(a) \phi(b) \phi(c) \text { for all } a, b, c \in N .
\end{aligned}
$$


Comparing both expressions, we obtain

$$
\theta(a)(\theta(b) d(c)+d(b) \phi(c))=\theta(a) \theta(b) d(c)+\theta(a) d(b) \phi(c) \text { for all } a, b, c \in N
$$

Since $\theta(N)=N$, it follows that

$$
a(\theta(b) d(c)+d(b) \phi(c))=a \theta(b) d(c)+a d(b) \phi(c) \text { for all } a, b, c \in N
$$

Lemma 2.3. Let $N$ be an arbitrary zero-symmetric nearring. If $d$ is a $(\theta, \phi)$-derivation on $N$ and $\theta(2 a)=2 \theta(a)$ for all $a \in N$, then $d(a b)=d(a) \phi(b)+\theta(a) d(b)$ for all $a, b \in N$.

Proof.

$$
\begin{aligned}
d((a+a) b)= & \theta(a+a) d(b)+d(a+a) \phi(b) \\
= & (\theta(a)+\theta(a)) d(b)+(d(a)+d(a)) \phi(b) \\
& \theta(a) d(b)+\theta(a) d(b)+d(a) \phi(b)+d(a) \phi(b)
\end{aligned}
$$

and

$$
d(a b+a b)=\theta(a) d(b)+d(a) \phi(b)+\theta(a) d(b)+d(a) \phi(b) .
$$

Equating the two expressions yields the desired result.

The proof of the next lemma is trivial.

Lemma 2.4. Let $N$ be a reduced nearring.

(i) If $a, b \in N$ and $a b=0$, then $b a=0$.

(ii) If $a, b \in N$ and $a b=0$, then $a N b=\{0\}$.

Lemma 2.5. Let $N$ be an arbitrary nearring. If $A$ is a nonzero semigroup ideal of $N$ and $d$ is a standard $(\theta, \phi)$-derivation on $N$ which acts as homomorphism on $A$, then

$$
(d(z)-\theta(z)) \theta(x) d(y)=0 \text { for all } x, y, z \in A .
$$

Proof. Since $d$ acts as a homomorphism on $A$, we have

$$
d(x y)=\theta(x) d(y)+d(x) \phi(y)=d(x) d(y) \text { for all } x, y \in A .
$$

Replacing $x$ by $z x$ in (2.1), we get

$$
\theta(z) \theta(x) d(y)+d(z) d(x) \phi(y)=d(z) d(x) d(y) \text { for all } x, y, z \in A \text {. }
$$

and it follows that

$$
\theta(z) \theta(x) d(y)+d(z) d(x) \phi(y)=d(z)(\theta(x) d(y)+d(x) \phi(y)) \text { for all } x, y, z \in A \text {. }
$$


Using Lemma 2.2, we find that

$$
\theta(z) \theta(x) d(y)+d(z) d(x) \phi(y)=d(z) \theta(x) d(y)+d(z) d(x) \phi(y) \text { for all } x, y, z \in A .
$$

i.e.

$$
\theta(z) \theta(x) d(y)=d(z) \theta(x) d(y) \text { for all } x, y, z \in A
$$

Thus,

$$
(d(z)-\theta(z)) \theta(x) d(y)=0 \text { for all } x, y, z \in A .
$$

Lemma 2.6. Let $N$ be an arbitrary nearring. If $A$ is a nonzero semigroup ideal of $N$ and $d$ is a standard $(\theta, \phi)$-derivation on $N$ which acts as an anti-homomorphism on $A$, then

$$
[\theta(z), d(y)] \theta(x) d(y)=0 \text { for all } x, y, z \in A .
$$

Proof. Let $d$ act as an anti-homomorphism on $A$. Then we have

$$
d(x y)=\theta(x) d(y)+d(x) \phi(y)=d(y) d(x) \text { for all } x, y \in A \text {. }
$$

Replacing $x$ by $x y$ in (2.2), we obtain

$$
\theta(x) \theta(y) d(y)+d(y) d(x) \phi(y)=d(y) d(x y) \text { for all } x, y \in A--
$$

i.e.

$$
\theta(x) \theta(y) d(y)+d(y) d(x) \phi(y)=d(y)(\theta(x) d(y)+d(x) \phi(y)) \text { for all } x, y \in A .
$$

Using Lemma 2.2, we get

$$
\theta(x) \theta(y) d(y)+d(y) d(x) \phi(y)=d(y) \theta(x) d(y)+d(y) d(x) \phi(y) \text { for all } x, y \in A,
$$

so that

$$
\theta(x) \theta(y) d(y)=d(y) \theta(x) d(y) \text { for all } x, y \in A .
$$

Replacing $x$ by $z x$ in (2.3), we find that

$$
\theta(z) \theta(x) \theta(y) d(y)=d(y) \theta(z) \theta(x) d(y) \text { for all } x, y, z \in A \text {. }
$$

Again using (2.3), the above relation gives that

$$
\begin{aligned}
& \theta(z) d(y) \theta(x) d(y)=d(y) \theta(z) \theta(x) d(y) \text { for all } x, y, z \in A . \\
& (\theta(z) d(y)-d(y) \theta(z)) \theta(x) d(y)=0 \text { for all } x, y, z \in A--
\end{aligned}
$$

i.e.

$$
[\theta(z), d(y)] \theta(x) d(y)=0 \text { for all } x, y, z \in A .
$$




\section{Main results}

Theorem 3.1. Let $N$ be 3-prime. Let $A$ be a nonzero semigroup ideal of $N$ such that $\theta(A)=A$ and $\phi(A)=A$. If $d$ is a standard $(\theta, \phi)$-derivation on $N$ which acts as a homomorphism or an anti-homomorphism on $A$, then $d=0$.

Proof. Assume first that $d$ act as a homomorphism on $A$. By Lemma 2.5, we have $(d(z)-$ $\theta(z)) \theta(x) d(y)=0$ for all $x, y, z \in A$; and since $\theta(A)=A$, we see that $(d(z)-\theta(z)) A d(y)=0$ for all $y, z \in A$. It follows by Lemma 2.1 (ii) that $d(z)-\theta(z)=0$ for all $z \in A$ or $d(A)=\{0\}$. In the latter case, using the definition of $(\theta, \phi)$-derivation and Lemma 2.1 (i), we get $d=0$. Thus, assume $d(z)-\theta(z)=0$ for all $z \in A$, in which case equation (2.1) implies $d(x) \phi(y)=0$ for all $x, y \in A$. Since $\phi(A)=A$, another appeal to Lemma 2.1 (i) gives $d(A)=\{0\}$ and hence $d=0$.

Assume now that $d$ acts as an anti-homomorphism on $A$. Since $\theta(A)=A$, Lemma 2.6 shows that $d(A)$ centralizes $A$; hence by Lemma 2.1 (iii) $d(A) \subseteq Z$. Thus, $d$ acts as a homomorphism.

We proceed now to the case of 3-semiprime nearrings.

Theorem 3.2. If $N$ is 3-semiprime and $d$ is a standard $(\theta, \theta)$-derivation which acts as a homomorphism on $N$, then $d=0$.

Proof. Using the same method in Lemma 2.5, we get

$$
(d(z)-\theta(z)) \theta(x) d(y)=0, \text { for all } x, y, z \in N .
$$

Replacing $z$ by $z w, w \in N$ in this equation, we have

$$
\begin{aligned}
0 & =(d(z w)-\theta(z w)) \theta(x) d(y)=(\theta(z) d(w)+d(z) \theta(w)-\theta(z) \theta(w)) \theta(x) d(y) \\
& =(\theta(z) d(w)+(d(z)-\theta(z)) \theta(w)) \theta(x) d(y) \\
& =\theta(z) d(w) \theta(x) d(y)+(d(z)-\theta(z)) \theta(w x) d(y) \\
& =\theta(z) d(w) \theta(x) d(y) \text { for all } x, y, z, w \in N
\end{aligned}
$$

Since $\theta(N)=N$ and $\theta(a b)=\theta(a) \theta(b)$ for all $a, b \in N$, we conclude that $\theta(x) d(y) N \theta(x) d(y)=0$ for all $x, y \in N$, so that $N d(y)=0$ for all $y \in N$. Since $N$ is 3-semiprime, we have $d=0$.

For reduced nearrings, which form a subclass of the class of 3-semiprime nearrings, we can get a result similar to Theorem 3.1 without the assumption that $\theta=\phi$.

Theorem 3.3. Let $N$ be reduced, and let $d$ be a standard $(\theta, \phi)$-derivation. Assume also that $\phi(N)=N$ and $\theta(2 a)=2 \theta($ a) for all $a \in N$. If d acts as a homomorphism or an anti-homomorphism on $N$, then $d=0$. 
Proof. Consider first $d$ acting as a homomorphism on $N$. In a right nearring, $-x y=(-x) y$ for all $x, y \in N$; hence Lemma 2.5 gives

$$
(d(z)-\theta(z)) \theta(x) d(y)=0=(\theta(z)-d(z)) \theta(x) d(y) \text { for all } x, y \in N .
$$

Replacing $z$ by $z w$ in the first equality and applying Lemma 2.3, we see that the left annihilator of $\theta(N) d(N)$ contains

$$
\begin{aligned}
d(z) \phi(w)+\theta(z) d(w)-\theta(z) \theta(w) & =d(z) \phi(w)+(\theta(z)-d(z)+d(z)) d(w)-\theta(z) \theta(w) \\
& =d(z) \phi(w)+(\theta(z)-d(z)) d(w)+d(z w)-\theta(z w)
\end{aligned}
$$

By (3.1) and Lemma 2.4 (ii), it follows that

$$
d(z) \phi(w) \theta(x) d(y)=0 \text { for all } x, y, z, w \in N .
$$

Postmultiplying by $\phi(u) \theta(v)$ and using the hypothesis that $N$ is reduced, we see that $d(N) N N$ $=\{0\}$; hence $d(N) d(N) d(N)=\{0\}$ and therefore $d(N)=\{0\}$.

Finally, suppose that $d$ acts as an anti-homomorphisms on $N$. By Lemma 2.6 we have

$$
[\theta(z), d(y)] \theta(x) d(y)=0 \text { for all } x, y, z \in N .
$$

so by Lemma 2.4

$$
d(y)[\theta(z), d(y)] \theta(x)=0=\theta(z) d(y)[\theta(z), d(y)] \theta(x)=d(y) \theta(z)[\theta(z), d(y)] \theta(x) .
$$

Since $\theta(N)=N$, we conclude that $[\theta(z), d(y)]^{2}=0$ for all $y, z \in N$, so that $d(N) \subseteq Z$ and therefore $d$ acts as a homomorphism.

\section{References}

[1] M. Ashraf, Ali Asma and Ali Shakir, $(\sigma, \tau)$-derivations on prime near rings, Arch. Math., 40(2004), $281-286$.

[2] A. Ali, N. Rehman and A. Shakir, On Lie ideals with derivations as homomorphisms and antihomomorphisms, Acta Math. Hungar., 101(2003), 79-82.

[3] H. E. Bell, On derivations in near rings II, Kluwer Academic Publ. Math. Appl. Dordr., 426(1997), $191-197$.

[4] H. E. Bell and L. C. Kappe, Rings in which derivations satisfy certain algebraic conditions, Acta. Math. Hungar., 53 (1989), 339-346.

[5] X. K. , Wang, Derivations in prme near rings, proc. Amer. Math. Soc., 121(1994), 361-366.

Department of Mathematics, Aligarh Muslim University, Aligarh 202002, India.

E-mail: asma_ali2@rediffmail.com

Department of Mathematics, Brock University, St.Catharines, Ontario L2S 3AI, Canada.

E-mail: hbell@brocku.ca

Department of Mathematics, N. R. E. C. Collage, Khurja - 203131, India.

E-mail: linerekha2@yahoo.co.in 